\title{
Giant Intranasal Pleomorphic Adenoma: A Rare Case Report
}

\author{
Shrinivas S Chavan, KS Bhople, Jeevan N Vedi, Prateek V Jain, Mangala Sonavani
}

\begin{abstract}
Pleomorphic adenoma, a heterogeneous mixture of epithelial and myoepithelial cells, commonly found in major salivary glands, accounts for about $3 \%$ of benign head and neck tumors. Intranasal pleomorphic adenoma (INPA) is an extremely rare variant forming a mere $8 \%$ of all pleomorphic adenomas. Majority of these arise from the nasal septum (82-90\%) with only about $8 \%$ showing attachment to the lateral nasal wall. Various theories explaining etiopathological basis of INPA have been proposed but none explains its predominance in females in 3 rd to 6 th decade of life. Hallmark of histopathological picture of pleomorphic adenoma is epithelial tissue mixed with tissues of myxoid, chondroid or mucoid origin. But INPA shows predominantly epithelial component with scanty stroma. Considering its high cellularity and anatomical site, INPA is often misdiagnosed as unilateral nasal malignancy. Early diagnosis ensures complete excision and is paramount to make patient disease free, considering its high potential of recurrence. Hereby, we are presenting a case of giant pleomorphic adenoma of nasal cavity, arising from lateral nasal wall, causing erosion of the base of skull, with the intent of drawing attention to this rare diagnosis.
\end{abstract}

Keywords: Giant intranasal pleomorphic adenoma, Lateral nasal wall attachment, Lateral rhinotomy approach.

How to cite this article: Chavan SS, Bhople KS, Vedi JN, Jain PV, Sonavani M. Giant Intranasal Pleomorphic Adenoma: A Rare Case Report. Clin Rhinol An Int J 2013;6(2):96-99.

Source of support: Nil

Conflict of interest: None declared

\section{INTRODUCTION}

Denker and Kahler in 1929 gave world the first reported case of intranasal pleomorphic adenoma (INPA) ${ }^{2}$ and since then approximately 150 cases of INPA have been reported in literature. ${ }^{1,3}$ Pleomorphic adenoma is defined as a benign heterogeneous tumor of salivary glands, composed of several tissue components of epithelial and myoepithelial origin. They are found most commonly in parotid gland, followed by submandibular gland, sublingual gland, palate. They are rarely seen in nasopharynx and nasal cavity with nasal septum being the most common site present in 82.5 to $90 \%$ patients of INPA while only $8 \%$ showing attachment to lateral nasal wall. INPA predominantly affect females, commonly in 3rd to 6th decade of their lives, the reason to which is still unexplained. Even after the advent of noninvasive preoperative diagnostic modalities like $\mathrm{CT}$ and magnetic resonance imaging (MRI), biopsy and histopathology still remains the gold standard diagnostic test. INPA shows predominantly epithelial component with scanty stroma as compared to their counterparts in major salivary glands. Considering its high cellularity and anatomical site, INPA is often misdiagnosed as unilateral nasal malignancy. Surgical resection is the treatment of choice. With advances in endoscopic surgery, most surgeons are opting for endoscopic removal for smaller masses. But the age old lateral rhinotomy approach still holds well, especially when the tumor mass is large. Here we present a case of a giant pleomorphic adenoma in a 65 years old female, showing attachment to lateral nasal wall, causing erosion of base of skull and occupying entire nasal cavity, deviating the nasal septum to opposite side.

\section{CASE REPORT}

A 65 years old female presented in Otorhinolaryngology Department with right side nasal blockade since 1 year and mass in right nostril since 2 months. She had h/o episodes of intermittent, unprovoked, fresh nasal bleed associated with epiphora, initially on right side that gradually became bilateral. Anterior rhinoscopy revealed a soft pinkish nonulcerated mass, filling the right nostril with DNS to left (Fig. 1). Probe could be passed all around except laterally. There was bilateral maxillary and ethmoidal tenderness. Posterior rhinoscopy revealed similar mass filling the nasopharynx. Contrast enhanced computed tomographic (CT) scan showed well-defined isodense mass of $86 \times 53 \times$ $45 \mathrm{~mm}$ in nasal cavity with displacement of nasal septum to left and smooth erosion of turbinates. The mass was extending anteriorly up to anterior nares, posteriorly up to nasopharynx and abutting skull base superiorly with bowing of bilateral lamina papyracea. There was smooth scalloping of maxillary sinuses, anterior ethmoidal cells, orbits and floor of the sphenoid sinuses and thinning of hard palate (Fig. 2). Considering the extent of mass, a lateral rhinotomy approach was planned. A papillomatous, grayish mass was seen in right nasal cavity extending up to sphenoid sinus and base of skull superiorly with attachment to the lateral nasal wall. There was erosion of perpendicular plate of ethmoid and vomer (Figs 3 and 4). On histopathological examination, there were strands of myoepithelial and epithelial cells surrounded by dense collagen and myxoid material and reported as pleomorphic adenoma (Figs 5 and 6). Patient recovered well postoperatively with healthy scar and showed no evidence of mass on endoscopic examination (Fig. 7). 


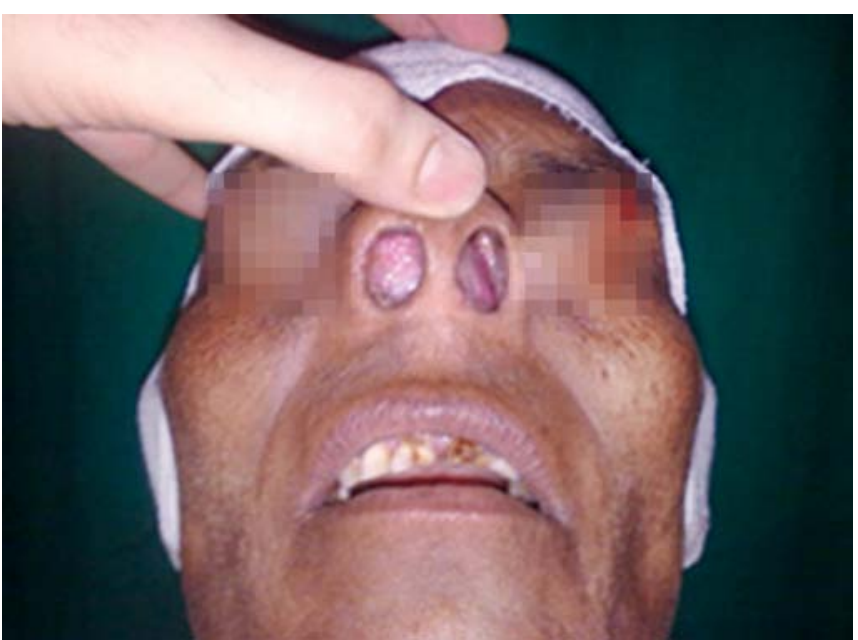

Fig. 1: Preoperative photograph of the patient showing pinkish nonulcerated mass, filling the right nostril deviating the nasal septum to left

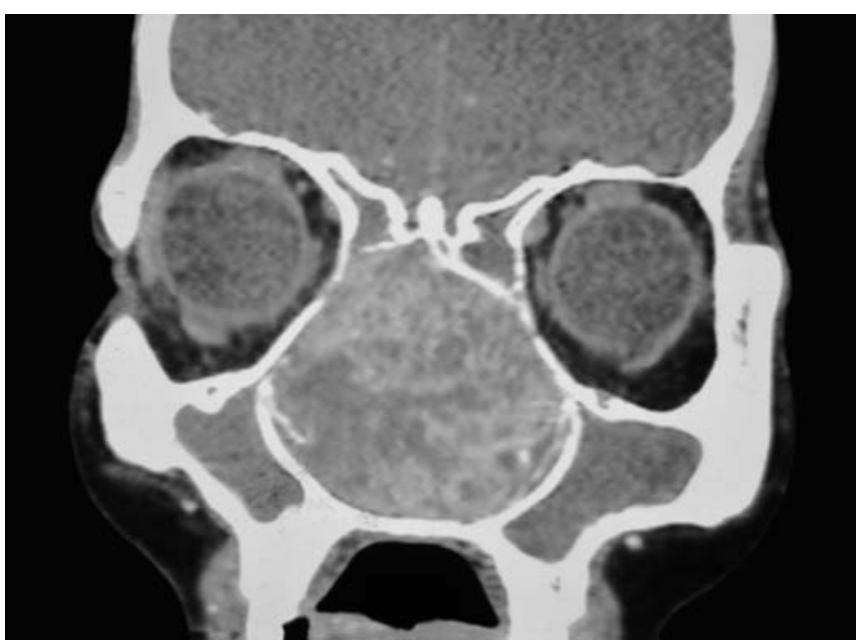

Fig. 2: CT scan showing well-defined isodense mass of $86 \times 53$ $\times 45 \mathrm{~mm}$ in nasal cavity with displacement of nasal septum to left and smooth erosion of turbinates, abutting skull base superiorly with bowing of bilateral lamina papyracea

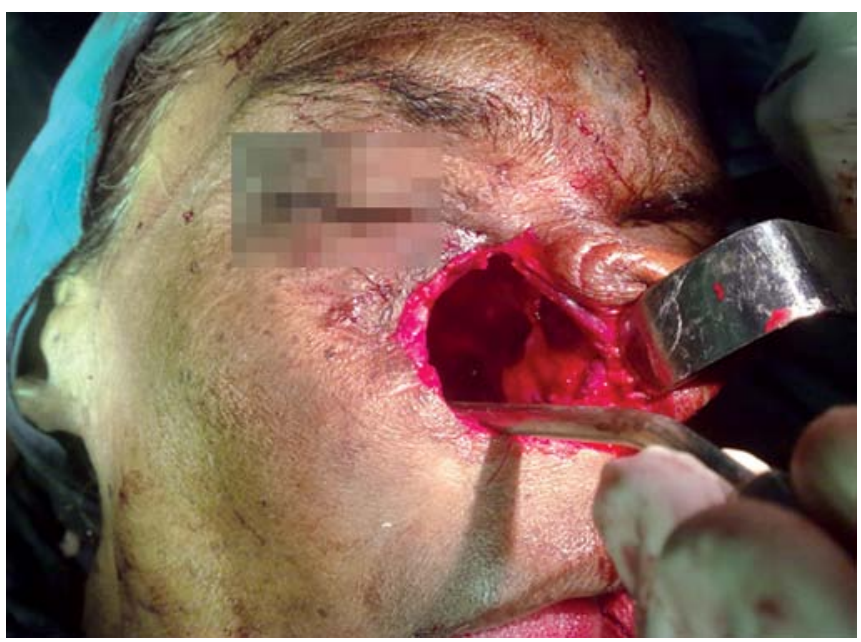

Fig. 3: Intraoperative photograph showing the cavity formed after removal of mass

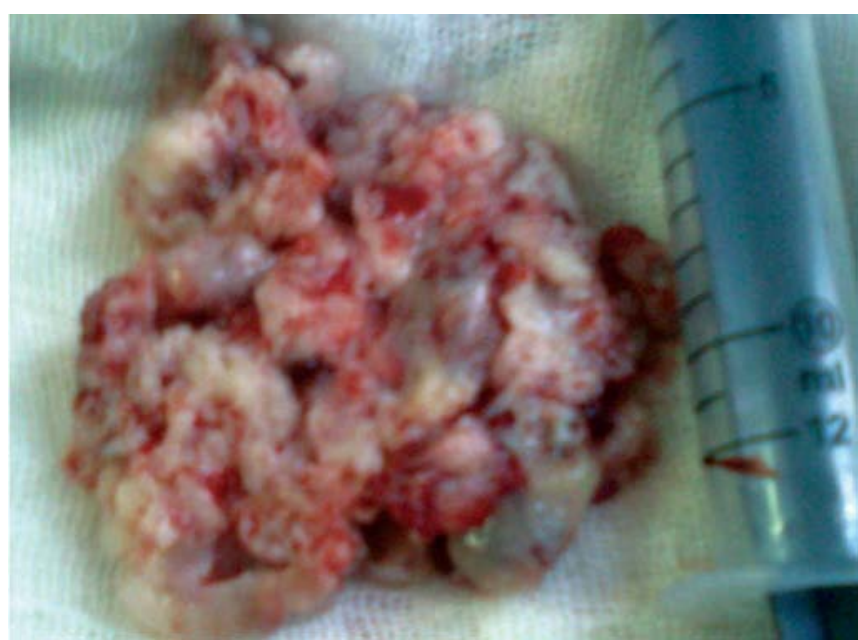

Fig. 4: Postoperative image of the mass removed in toto

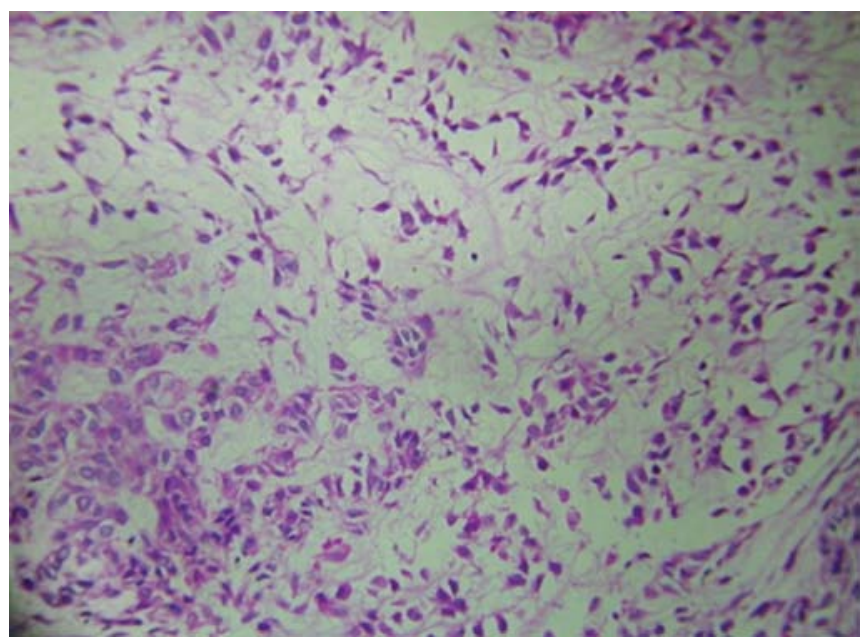

Fig. 5: Histopathological picture on hematoxylin and eosin (H\&E) staining showing strands of myoepithelial and epithelial cells surrounded by dense collagen and myxoid material (magnification 40x)

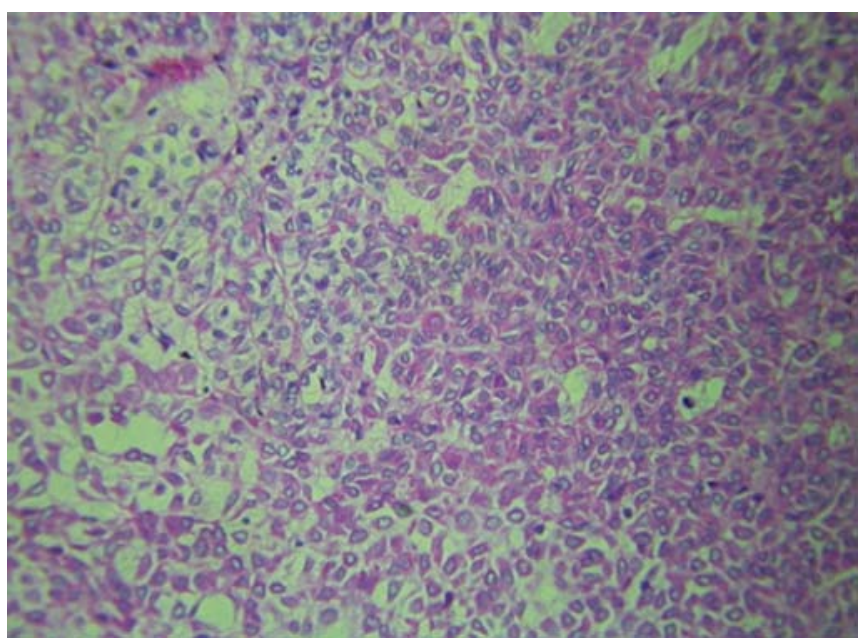

Fig. 6: Histopathological picture on H\&E staining showing excessive cellularity and scanty stroma with few keratin pearls which can be confused with squamous cell carcinoma (magnification 20x) 


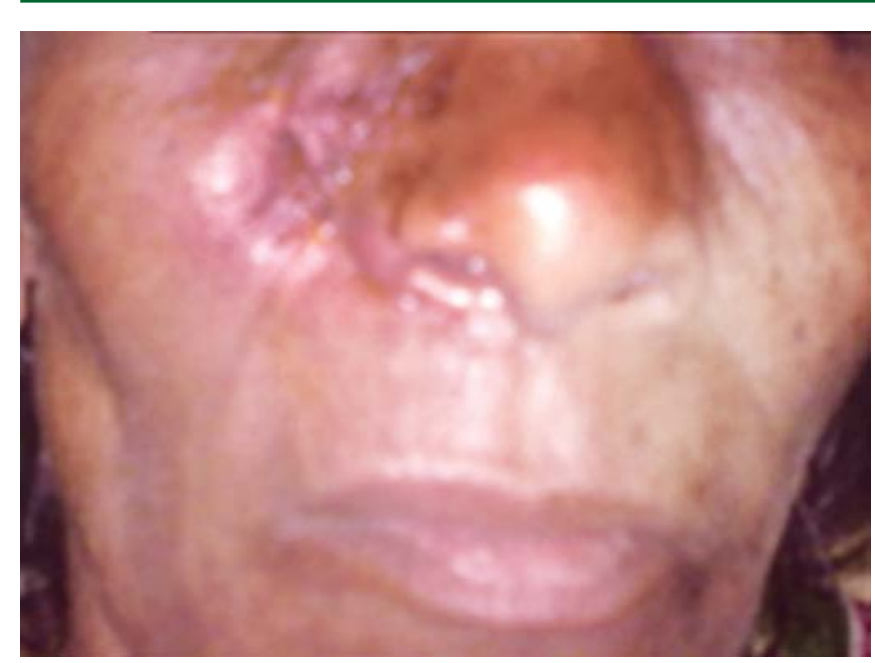

Fig. 7: Postoperative photograph of patient on day 10 showing healed scar

\section{DISCUSSION}

Salivary gland tumors constitute about $3 \%$ of all neoplasms. The majority of these tumors are benign and about $70 \%$ are pleomorphic adenomas. A small minority (8\%) are located in the oral cavity, neck and nasal cavity. INPA generally arises in the nasal septal mucosa $(82.5-90 \%)$ and rarely shows attachment to lateral nasal wall $(8 \%)$, even though the seromucosal glands are mainly located within the lateral nasal wall. ${ }^{1}$

Stevenson suggested that remnants of the vomeronasal organ, an epithelium-lined duct in the cartilaginous nasal septum degenerated in early fetus, could be the reason for the appearance of these tumors in the nasal turbinate. ${ }^{4}$ Whereas, Ersner and Saltzman postulated that the precursors of the septal pleomorphic adenomas are ectopic embryonic epithelialized cells on the nasal septum mucosa, found during the migration of the nasal buds. ${ }^{5}$ According to Evans and Cruikshank, it originates directly from the matured salivary glandular tissue. ${ }^{6}$ Dawe, in 1979, proposed a viral etiology from polyomavirus. ${ }^{7}$

Nasal pleomorphic adenoma is seen predominantly in females in 3rd to 6th decade of their life. ${ }^{8}$ None of the present theories for etiology of INPA provide any insights to this female predilection. A genetic study may be indicated to search for this missing link in the etiopathogenesis of INPA.

Known to be a slow growing tumor, the clinical features such as unilateral nasal blockade and epistaxis usually appear after a long silent period. It appears as unilateral, smooth surfaced, grayish-white, easily bleeding, nonulcerated mass on rhinoscopy.

MRI is a better noninvasive diagnostic modality as compared to CT for INPA, as it is possible to assess its relationship with adjacent structures in several anatomic planes. ${ }^{9} \mathrm{~T} 1 \mathrm{~W}$ MRI shows low to intermediate signal intensity and T2W MRI shows intermediate to high density signals. A hypointense capsule may be visible on T2W images. On contrast enhanced images, it exhibits heterogeneous enhancement. Nonenhanced CT paranasal sinus reveals a well defined, lobulated mass displacing the nasal septum as in this case and is useful to show calcifications.

Wide local excision is generally agreed as the treatment of choice. Lateral rhinotomy, midfacial degloving, transpalatal surgery or endoscopic resection are various approaches used for surgery. ${ }^{10}$ Lateral rhinotomy was planned in this case considering the extent of the disease. The external approach via lateral rhinotomy should not be a deterrent as good cosmetic outcome can be achieved. Postoperative radiotherapy has been advocated by some authors in circumstances where residual disease or intracranial extension is present.

Histologically, INPA is characterized by epithelial tissue mixed with tissues of myxoid, chondroid or mucoid origin. It may resemble aggressive epithelial tumors because of high cellularity and lack of stromal component when compared to their counterparts at other sites. ${ }^{11}$

These tumors have a potential to recur. The recurrent lesions constitute more stroma than cellular elements and spillage of the former in the surgical field is thought to provide the nidus for recurrence. ${ }^{5}$ Rarely malignant transformation occurs with carcinoma ex-pleomorphic adenoma being the commonest variant. It has the potential to metastasize with bone being the predominant site. ${ }^{3}$

Given the rarity of the INPA and heterogeneity of its behavior, no standard treatment algorithm has been established. Nevertheless, postoperative follow-up examination is mandatory with its potential for recurrence.

\section{CONCLUSION}

Nasal cavity is a rare site for pleomorphic adenoma, with nasal septum being the commonest site of attachment for INPA. Only $8 \%$ cases of INPA originate from lateral nasal wall abutting the inferior and middle turbinate.

INPA shows predilection toward females in 3rd to 6th decade, reason for which is still elusive. Present theories of origin of INPA fail to give conclusive reasoning for this. A further genetic study is indicated to better understand the etiology of INPA.

MRI is the preferred noninvasive diagnostic modality whereas histopathological examination is the gold standard investigation for diagnosing INPA.

INPA differs from the pleomorphic adenoma at other sites such as parotid gland on histopathological appearance 
in having more epithelial component and relatively less myxoid stromata.

Considering its high cellularity and anatomical site, INPA is often misdiagnosed as unilateral malignancy.

Recurrent INPA show more myxoid tissue and stromal element, spillage of which in the surgical field is thought to provide nidus for recurrence.

Giant INPA are excised by external approach, providing better exposure. Midfacial degloving, transpalatal approach and endoscopic removal are other approaches used. Radiotherapy is considered in cases where incomplete removal of tumor or intracranial extension is suspected.

Early diagnosis offers the possibility of a more complete excision with adequate care being taken not to disrupt the tumor in order to prevent local and distant spread of neoplastic cells. Long term follow-up, both endoscopic and radiologic, to exclude malignancy is mandatory, even if the tumor appears to be clinically benign and resected completely.

\section{REFERENCES}

1. Jassar P, Stafford ND, MacDonald AW. Pleomorphic adenoma of the nasal septum. J Laryngol Otol 1999 May;113:483-485.

2. Denker A, Kahler O. Handush der Hals. Nasen ohrenheilkunde 1929;5:202.

3. Freeman SR, Sloan P, de Carpentier J. Carcinoma ex-pleomorphic adenoma of the nasal septum with adenoid cystic and squamous carcinomatous differentiation. Rhinology 2003 Jun;41(2):118121.

4. Stevenson HN. Mixed tumor of the septum. Ann Otol Rhinol 1932;41:563-570.

5. Matthew S, Ersner MD, Saltzmann M. A mixed tumor of the nasal septum. Report of a case. Laryngoscope 1944;54(6):287-296.

6. Evans RW, Cruickshank AH. Epithelial tumors of the salivary glands. Major problems in pathology. Philadelphia, PA: WB Saunders Co; 1970. 167 p.
7. Dawe CJ. Tumors of salivary and lacrimal glands, nasal fossa and maxillary sinus. IASC Sci Publ 1979;23:91-113.

8. Motoori K, Takano H, Nakano K, Yamamoto S, Ueda T, Ikeda M. Pleomorphic adenoma of nasal septum: MR features. Am J Neuroradiol 2000 Nov-Dec;21(10):1948-1950.

9. Haque F, Babu SPH, Ahamed S, Ahmad I, Abbas SZ. Pleomorphic adenoma of nasal septum. Indian J Radiol Imag 2005 Aug; 15(3):311-313.

10. Karakus MF, Ozcan KM, Dere H. Endoscopic resection of pleomorphic adenoma of the nasal septum. Tumori 2007 MayJun;93(3):300-301.

11. Castello E, Caligo G, Pallestrini EA. Su un caso di adenoma pleomorfo della fossa nasale. Acta Otorhinolaryngol Ital 1996 Oct;16:433-437.

\section{ABOUT THE AUTHORS}

\section{Shrinivas S Chavan (Corresponding Author)}

Associate Professor, Department of Otorhinolaryngology, Government Medical College, Aurangabad, Maharashtra, India, e-mail: shrinivasc77@hotmail.com

\section{KS Bhople}

Dean, Department of Pathology, Government Medical College Aurangabad, Maharashtra, India

\section{Jeevan $\mathbf{N}$ Vedi}

Professor and Head, Department of Otorhinolaryngology, Government Medical College, Aurangabad, Maharashtra, India

\section{Prateek V Jain}

Resident, Department of Otorhinolaryngology, Government Medical College, Aurangabad, Maharashtra, India

\section{Mangala Sonavani}

Professor and Head, Department of Medicine, Government Medical College, Aurangabad, Maharashtra, India 